Brit. J. industr. Med., 1949, 6, 91.

\title{
CHANGES IN THE LUNGS PRODUCED BY NATURAL GRAPHITE
}

\author{
BY \\ H. E. HARDING and G. B. OLIVER* \\ From the Department of Pathology, Sheffield University \\ (RECEIVED FOR PUBLICATION, SEPTEMBER 1, 1948)
}

Graphite or plumbago is a crystalline form of carbon which occurs in nature as soft black masses or, less commonly, as crystals in igneous rocks : this natural graphite contains varying proportions of other minerals. Graphite is also made artificially from coke, and the best preparations of this kind are nearly pure carbon. This paper will deal with some of the effects of natural graphite, and the term "graphite" will be used to mean " natural graphite," that is, a mixture of minerals, largely crystalline carbon.

The main sources of graphite in 1935 were the U.S.S.R. 39 per cent., Korea 21 per cent., Germany 10 per cent., Austria 9 per cent., Ceylon 7 per cent., Madagascar 5 per cent., and Mexico 3 per cent. (Jones, 1943). During recent years the graphite used in Great Britain has come mainly from Ceylon and South-west Africa, but there has also been a small quantity mined in Borrowdale, Cumberland.

Not much more than 10 per cent. of the graphite produced in the world is used in pencils and crayons ; more than half of it is employed in foundries and in making crucibles for metallurgical purposes. Finely ground graphite is used extensively as a lubricant, either alone or in combination with oil or grease : considerable quantities of the poorer quality graphite are used in paints and pigments for protecting metallic surfaces from various corroding substances-for example, stove polish, which consists of graphite mixed with some amorphous carbon and a little grease. Graphite is also used for making electrodes and dynamo brushes, and in dry batteries.

For most purposes graphite has to be ground to a fine powder, and there is a small industry devoted to grinding graphite which it supplies to commercial users. In other cases the grinding is done as a

\footnotetext{
* Recently H.M. Medical Inspector of Factories.
}

subsidiary process by firms who themselves use large quantities of crushed graphite. The method of grinding varies according to the fineness of the powder desired. In the past the general practice was to grind the graphite in an open-edge runner mill or in a ball mill, and this method is still in use in some factories where extreme fineness is not required. The more modern method of grinding is in a totally enclosed mill with automatic feeding and discharge. The older methods of grinding, mixing, and bagging graphite produced very large quantities of dust which floated in the air and reduced visibility often to a few feet, but there is relatively little dust in the few modern factories. It is the purpose of this paper to show that serious damage to the lungs can and does result from the inhalation of this dust, and to suggest that the minerals other than carbon found in it are largely responsible for these effects.

The mining of graphite, at any rate in Ceylon, seems to carry only a very small risk of pulmonary damage (Dassanayake, 1948) since the drilling is usually done wet, but radiographic changes without physical disability have been detected in some miners who have worked underground for more than, twenty years. Radiographic changes are found earlier, are more marked, and tend to be associated with physical signs in workpeople engaged in curing graphite in Colombo.

Koopmann (1924) described the lungs of a man who had worked for fifty years in a graphite mill, and who was killed by accident. The lungs showed pneumoconiosis and cavities due to necrosis ; there was chronic interstitial pneumonia, peribronchiolitis, and a nodular type of arteritis. This man, however, had had periods of employment as a stonegrinder, and this history, together with the fact that Koopmann saw nothing to make him suspect silicosis, led to little notice being taken of the 
report. The marked radiological changes that can be found in grinders and mixers of graphite were demonstrated by Dunner (1945) and by Dunner and Bagnall (1946). Dunner recorded his findings in five men, all from the same works, who had been handling graphite in the manufacture of stove polish for from seventeen to thirty-four years, and whose films showed reticulation and nodulation in varying degree, and in some cases massive shadows. One of these men (Case 2) showed a massive reniform shadow in the right upper zone and two large fainter shadows in the left lung: this case was again recorded by Dunner and Bagnall in more detail after the patient had developed a distinct cavitation of irregular shape within the opacity in the left upper zone of his lungs. This man has since died, and a description of his lungs will be given in this paper together with some details of another workman from the same factory who died in 1932, and a report on the lungs of a man who had been employed for over twenty years in grinding graphite, mainly from Ceylon, for another firm. The graphite to which the first two workmen had been exposed was mainly Korean.

\section{Composition of Graphite}

In his original paper Dunner was misled by the statement of the workmen that they used only "pure graphite" into believing that crystalline carbon might be responsible for the changes; in fact the graphite was "pure" only in the sense that it was not adulterated after being mined. This error was recognized in the later paper of Dunner and Bagnall, who gave some figures of the composition of a finished product from the man's works.

Three samples of Ceylon graphite and one each from South-west Africa and Korea have been supplied by the firm, together with a sample of " carbon black" which is mixed with the graphite in the final product. The ash after incineration at $600^{\circ}$ C. ranged from $12 \cdot 81$ to 33.26 per cent. of the various graphites, but was only 0.34 per cent. of the carbon black. The graphites contained from 8.0 to 18.6 per cent. total silica, 3.6 to 10.0 per cent. free silica, and 0.8 to 4.5 per cent. iron. Analyses by $x$ rays gave figures for crystalline silica slightly greater than those found by chemical methods. It is evident, then, that graphite is a very variable mineral only two-thirds of which may be carbon, and that it contains, among other impurities, notable quantities of crystalline silica.

\section{Case Records}

Case 1.-J.T. died in 1932 when 58 years of age. He had for sixteen years been employed in emptying $1 \frac{1}{2}$ cwt. sacks of black lead into a mechanical mixer from which the powder was shovelled into a rotary sieve. His foreman agreed that the work was very dusty, but denied that any other workmen suffered any disability. He had not worked for fourteen months before his death and had been confined to bed most of this time. His doctor said that he had a catarrhal condition of his lungs with a severe cough, which later became very severe and was accompanied by a large quantity of very black sputum. Post-mortem examination showed both lungs bound to the chest wall and extensively fibrosed : there was no evidence of tuberculosis or of bronchiectasis. The cause of death was given at the inquest as " heart failure from fibrosis of the lungs through inhaling a black substance while following his occupation" (Hull Daily Mail, Sept. 27, 1932). As far as can be ascertained this was the first report of a post-mortem examination of a graphite worker in Great Britain.

By the courtesy of Dr. J. C. Lees, histologist to the Royal College of Physicians of Edinburgh, $I$ have been able to examine four blocks of tissue from this man's lungs. The sections show intense anthracosis. Two of the blocks show marked focal emphysema (fig. 1), while the other two show very considerable fibrosis, both diffuse and in the form of nodules that are highly suggestive of silicosis (fig. 2). There is peribronchial and perivascular fibrosis: the latter involves the wall of several vessels in which there is also considerable intimal thickening. There is diffuse black pigmentation throughout the densely fibrous areas, but less in the nodules: phagocytic cells filled with pigment also crowd some alveoli and plug some of the smaller bronchi. There is some purulent bronchitis, but no evidence of tuberculosis. Most of the black pigment has disappeared in incinerated sections but a small proportion of it is obviously iron: there is a fairly marked dusting with refractile silica particles throughout the fibrous areas.

Case 2 (This is the man whose radiographs were described by Dunner).-H.D. aged 67 years, was employed as a barman until he joined the Army in 1902. On leaving the Army in 1920 he worked on a graphite mill grinding Korean graphite until 1939, when he became unfit and was given light work involving no dust hazard. In 1947 he had lost over 2 stone in weight, and suffered from marked dyspnœa : he had very little sputum and not much cough. There was dullness to percussion in the right sub-clavicular region. A radiograph of his chest showed a massive homogeneous opacity in the right upper zone, a small rounded opacity overlying the lower part of the root of the right lung, a homogeneous opacity of less density in the left upper zone, a rounded opacity the size of a golf ball overlying the left root and the left cardiac border, and a fine mottling in the mid-zones of both lungs.

He died on April 9, 1948, and an autopsy was performed on behalf of the coroner by Dr. N. T. Whitehead. There were some adhesions between the upper parts of each lung and the chest wall. Small collections of black pigment, similar to graphite, were to be seen in the pleural lining of the chest wall : this pigment was also found throughout the lungs, which were jet black. On palpation there was a firm fibrous mass in each of the 


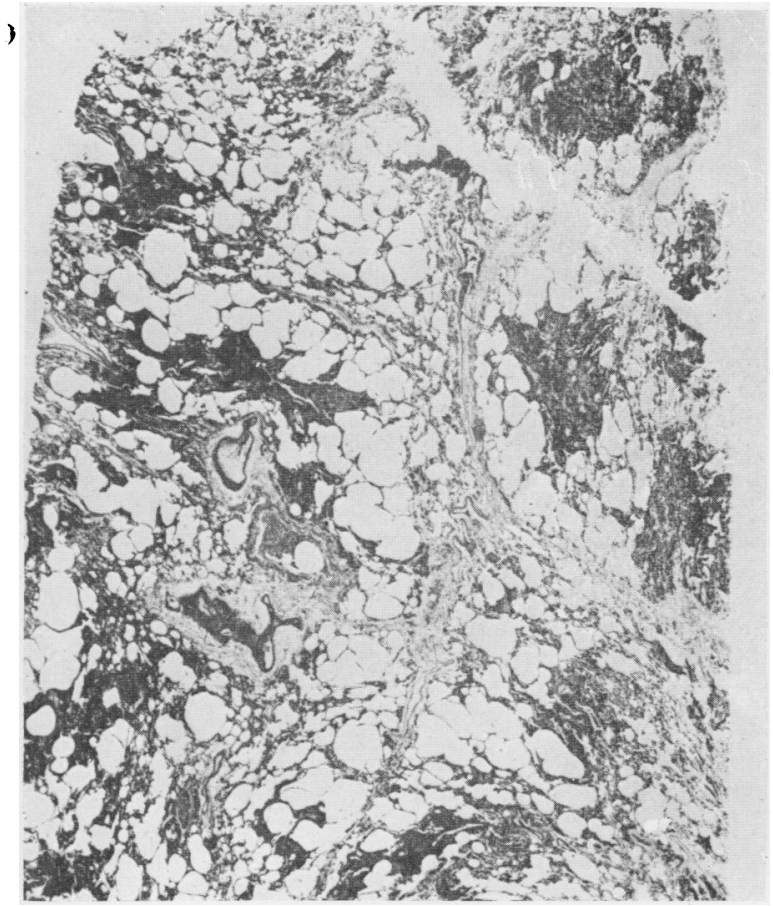

FIG. 1

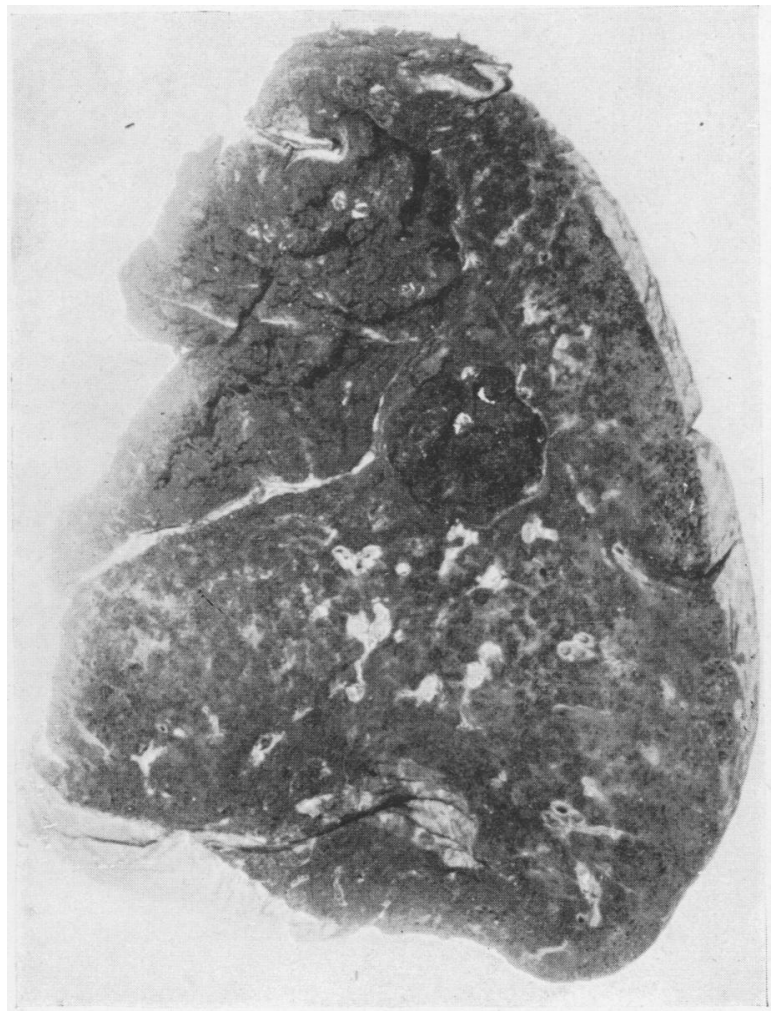

FIG. 3

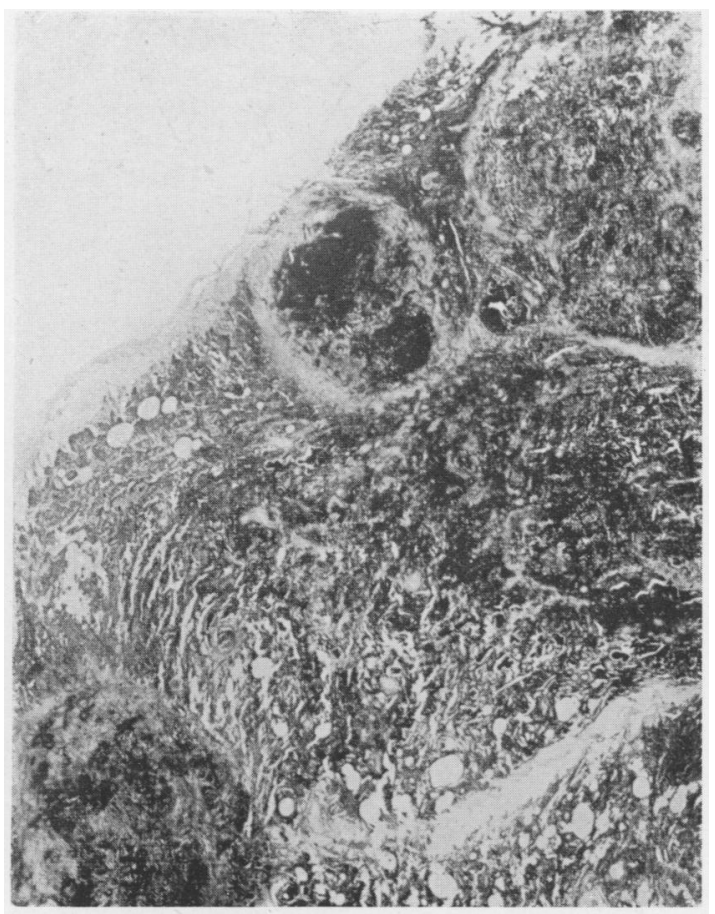

FIG. 2

FIG. 1.-Focal emphysema around pigment aggregates. Case $1 . \times 5$.

FIG. 2.-Silicotic nodules and diffuse dust fibrosis. Case $1 . \times 5$.

Fig. 3.-Right lung of Case 2. $\times \frac{1}{2}$. 


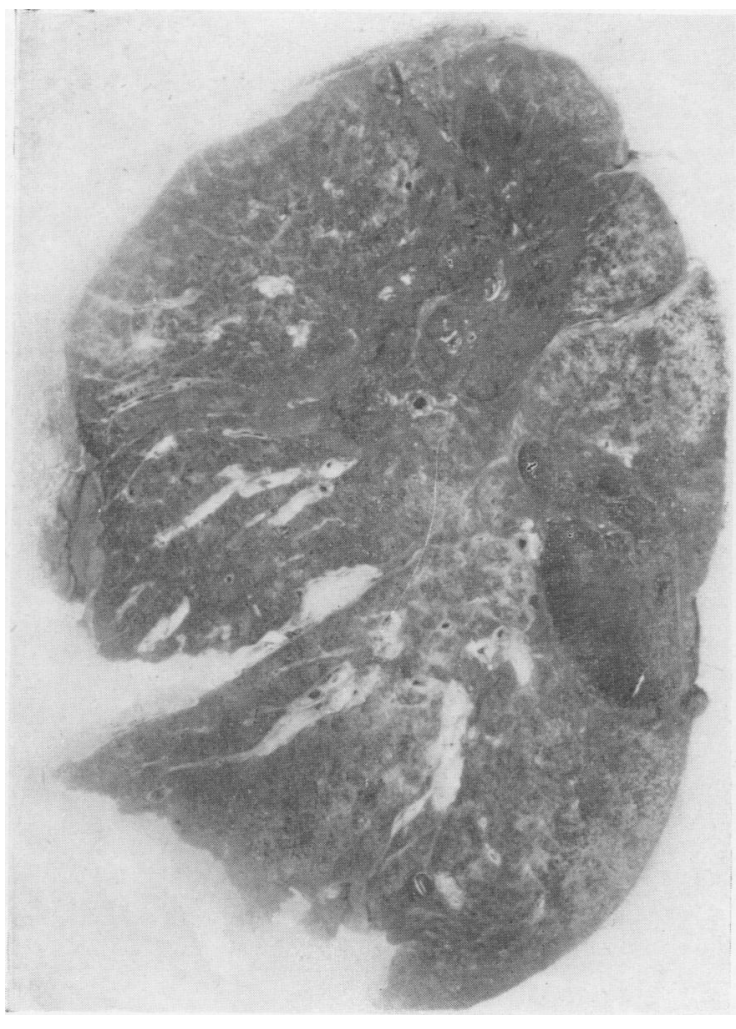

Fig. 4.-Left lung of Case 2, $\times \frac{1}{2}$. This and Fig. 3 are deliberately light prints in order to show detail; the lungs are much darker than the photographs suggest.

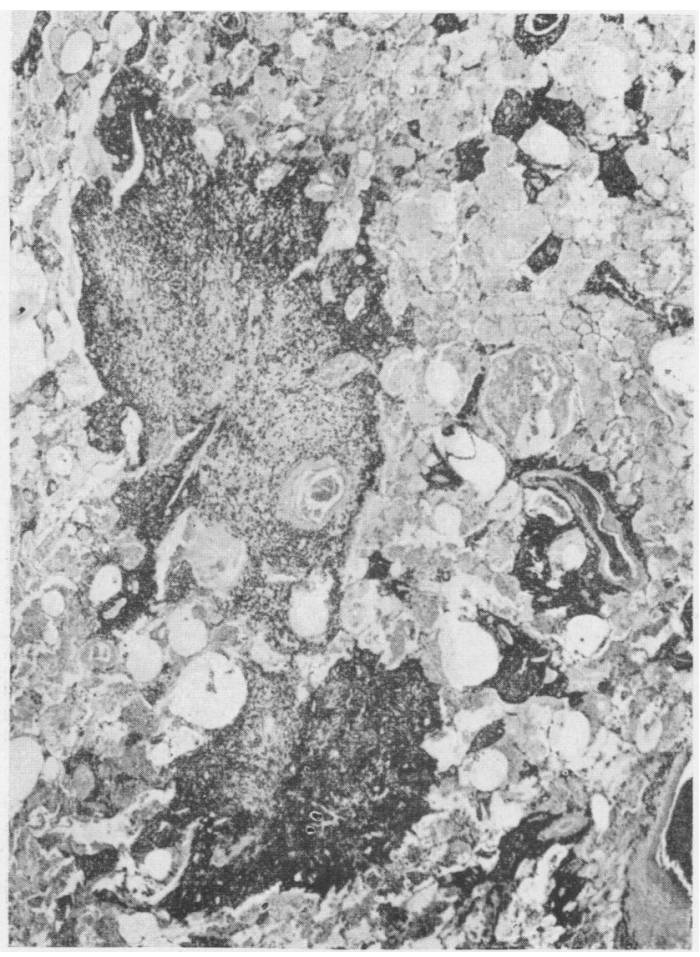

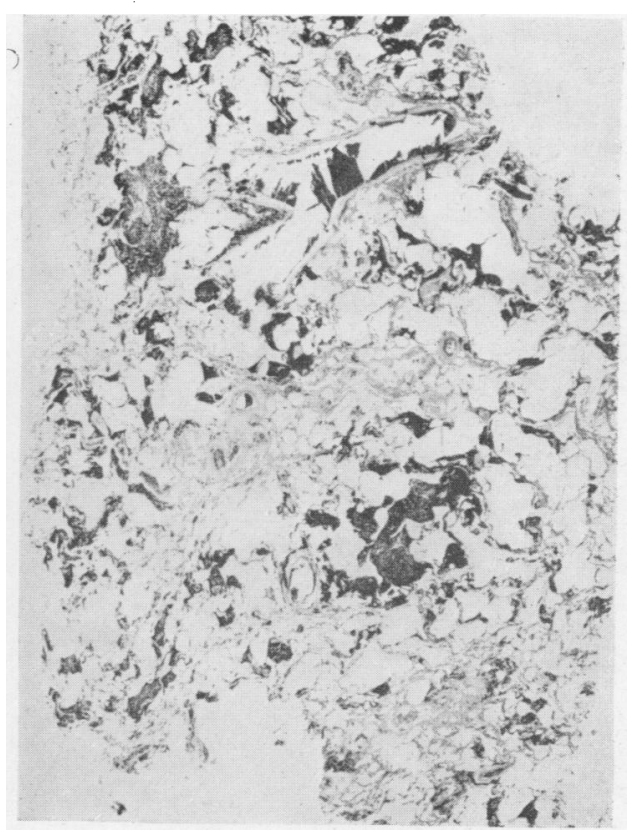

Fig. 5.-Focal emphysema around pigment aggregates. Case 2. $\times 5$.

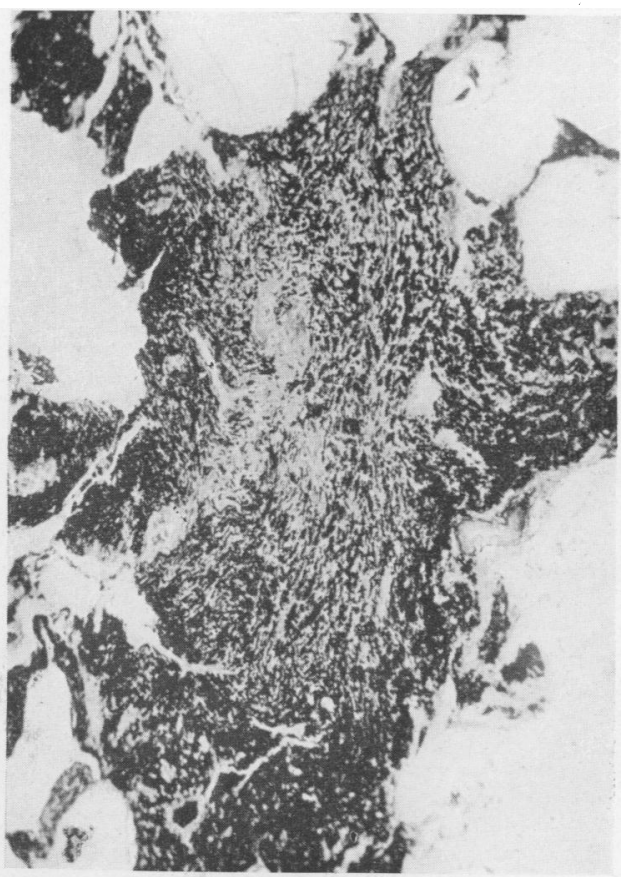

FIG. 7 (above).--Linear dust fibrosis. Case 2. $\times 32$.

Fig. 6 (left).-Protein precipitate in alveoli. Dust fibrosis. Case $2 . \times 10$. 


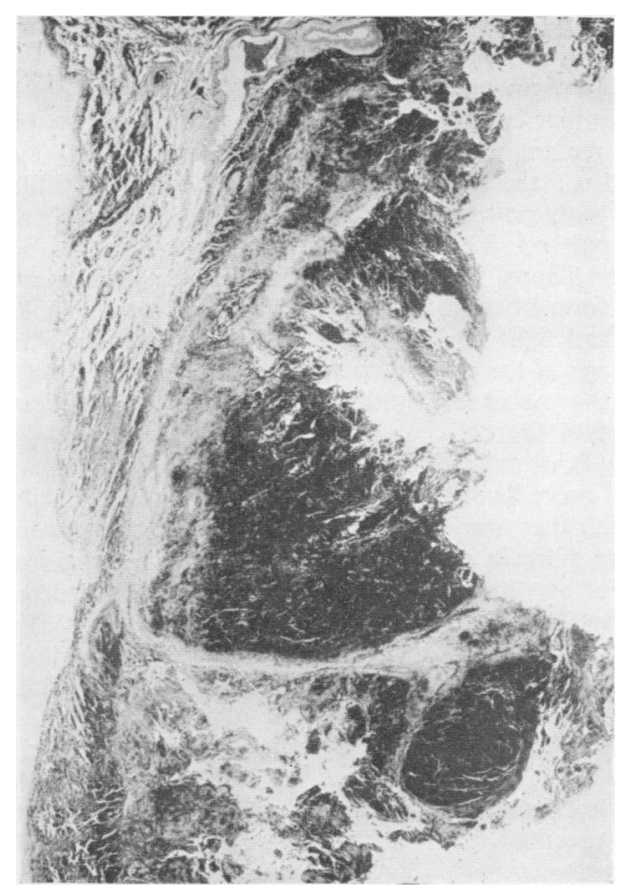

Fig. 8.-Border of large cavity. Case 2. $\times 5$.

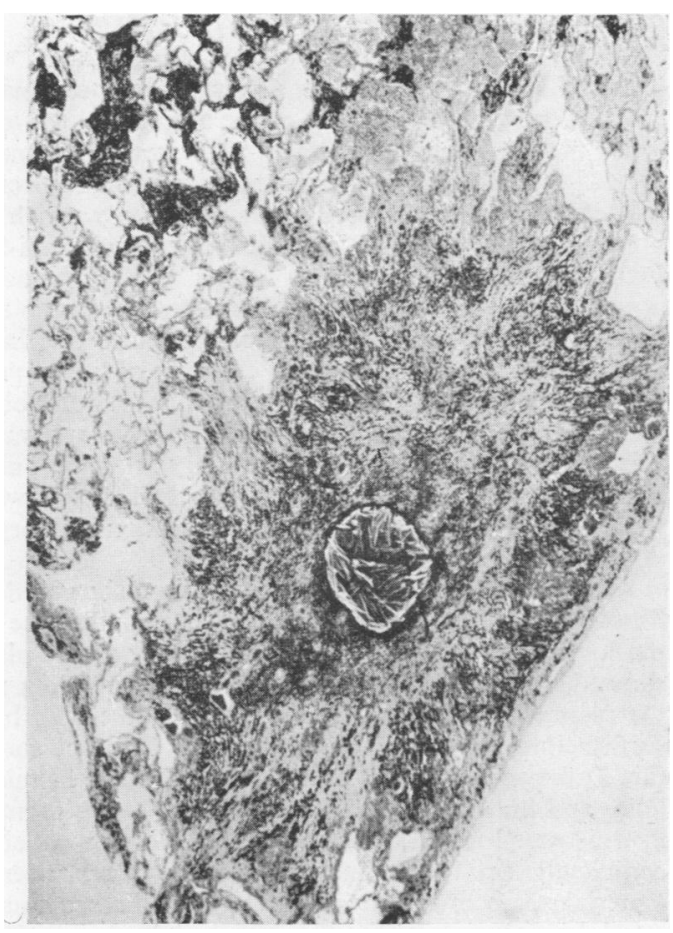

FIG. 9.-Early necrosis in the middle of a medusahead area of dust fibrosis. Case $2 . \times 10$.
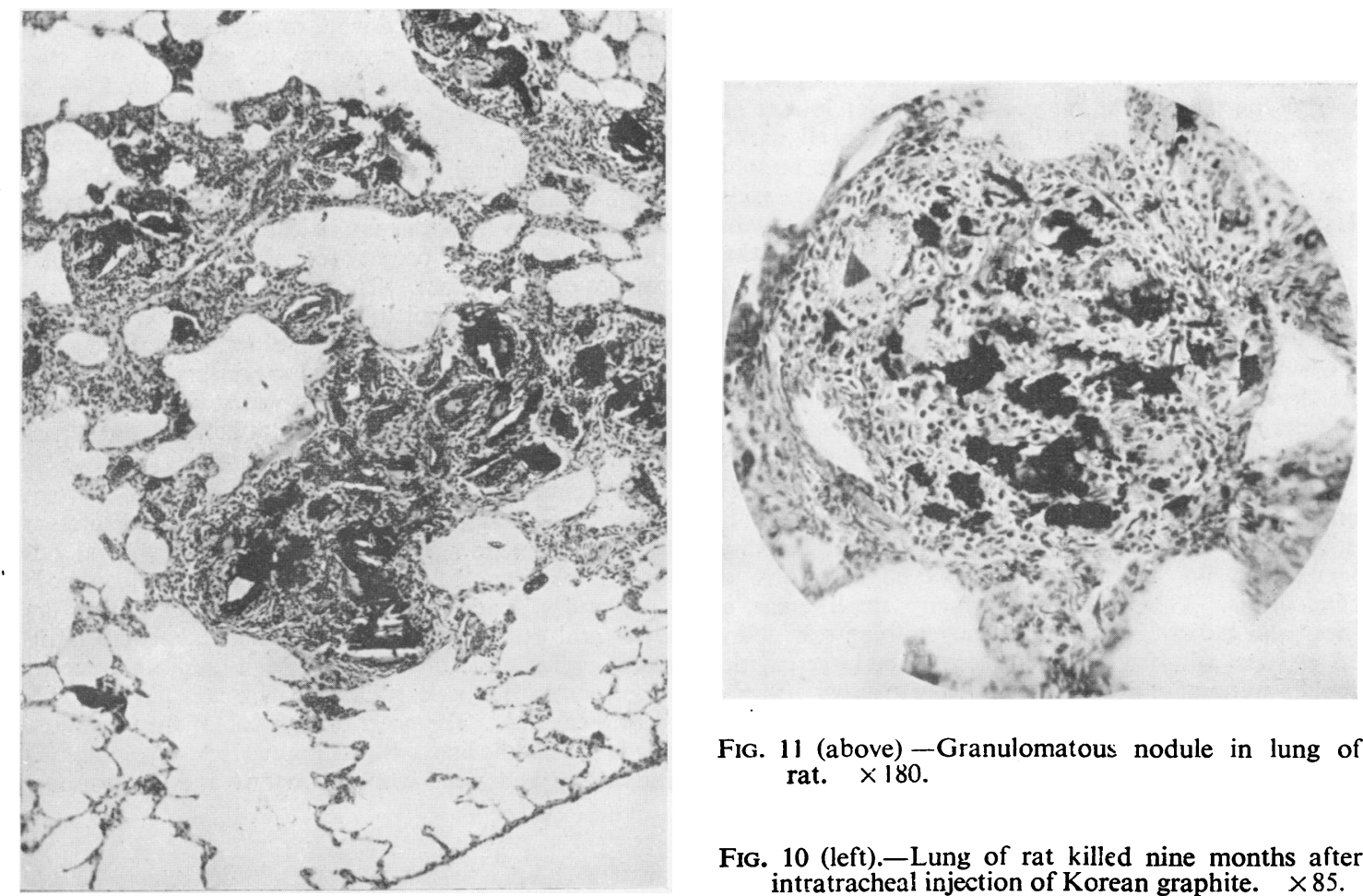

FIG. 11 (above) -Granulomatous nodule in lung of rat. $\times 180$.

Fig. 10 (left).-Lung of rat killed nine months after intratracheal injection of Korean graphite. $\times 85$. 
lobes of the lungs: in the upper lobes these masses reached to within two inches of the apices : in the lower lobes they were more central : all were approximately $2 \frac{1}{2} \times 2 \frac{1}{2} \times 3$ inches. In the centre of each mass necrosis had occurred with the production of a cavity which had a black ragged wall and was filled with black semi-fluid material. There were also three fibrotic nodules approximately half an inch in diameter, one in the right upper lobe, one in the right lower lobe, and one in the left lower lobe. There was no evidence of tuberculosis. Between the osophagus and the trachea were black, moderately enlarged lymph nodes. The heart muscle was soft and flabby. The left coronary artery was much narrowed by atheroma. Apart from the kidneys, which showed atherosclerotic changes, the abdominal organs and the brain appeared normal.

After fixation in formalin the right lung showed dense fibrous thickening of the pleura over the middle lobe and the lower part of the upper lobe, the rest of the pleura showed raised white lines, evidently corresponding with the pleural lymphatics, dividing up the otherwise black surface into irregularly shaped areas. The cut surface (fig. 3) showed that most of the upper lobe, all the middle lobe, and an area approximately $1 \frac{1}{2}$ inches in diameter in the lower lobe adjoining the middle lobe, were either completely solid or consisted of dull black necrotic material some of which had fallen out. There was no interlobar pleural space, the middle lobe being recognizable only from the grey-white bands of fibrous tissue representing the original pleura. The cavities containing necrotic material were bounded in much of their circumference by a thin band of light grey fibrous tissue ; elsewhere they abutted on dull black completely solid areas in which no fibrous tissue could be seen by the naked eye. Within the necrotic areas were irregularly shaped small portions of white tissue that represented the remains of pulmonary vessels. The rest of the lung showed numerous areas of dull black pigmentation which tended to run together in the middle of the lower lobe. The more peripheral pigmented areas showed well-marked focal emphysema but the central ones tended to be solid. The left lung (fig. 4) was essentially similar to the right. The large cavity in the lower lobe had a somewhat thicker and more complete grey fibrous capsule than those elsewhere. In the apex and posterior portion of this lower lobe it was possible to see bronchopneumonic exudate.

Chemical examination of the lungs showed that, of the dry weight, ash formed 5.65 per cent., total silica 1.14 per cent., free silica (method of Trostel and Wynne, 1940) 0.56 per cent., and iron (as $\mathrm{Fe}_{2} \mathrm{O}_{3}$ ) 0.98 per cent.

Histological examination showed widespread focal emphysema (fig. 5) which was somewhat masked by œdema (fig. 6), some purulent bronchitis and bronchopneumonia, but nothing that was even suggestive of tuberculosis. There were very many small areas of linear and radial fibrosis (fig. 7) but nothing resembling the classical whorled silicotic lesion. The large cavities (fig. 8) were bounded by a relatively narrow zone of dense fibrous tissue within which was an irregular wider zone of fibrosis so densely pigmented that it was almost impossible to make out its structure : inside this zone was deeply pigmented necrotic material that was mainly finely granular but showed also larger black particles of irregular rectangular shape and of the order of 25 to $40 \mu$ by 2 to $4 \mu$ : these were too black to show any possible staining with potassium ferrocyanide and $\mathrm{HCl}$; they disappeared on incineration, and were taken to be graphite. There were also present some colourless crystals, found best in frozen sections, that were visible in polarized light and appeared to be cholesterol. An earlier stage of the necrosis is seen in fig. 9, which shows some of the spaces left by these crystals. In the earliest stages found the collagen fibrils in small areas showed only very faint staining with either eosin or van Gieson and were more swollen than the normally stained fibrils with which they were continuous. Vascular changes in the larger fibrotic areas were easily found in sections stained for elastin : they were similar to those pictured by Stewart and Faulds (1934) and by Geever (1947) : many of them had progressed to complete obliteration of the lumen of arteries. Black pigment was widely distributed in large amounts, a little of it in phagocytes within alveoli, more within alveolar walls, and much more within the areas of fibrosis. Incinerated sections showed that most of this pigment was combustible, but a small proportion of it was iron. Incinerated and acid-treated sections showed a conspicuous dusting with refractive silica particles within the areas of fibrosis.

Case 3.-C.W., aged 71 years, up to the age of 40 had worked as a shoemaker. He then obtained employment grinding graphite (Ceylon and Madagascar) in an openedge runner mill for five years. Then followed a break of three years when he worked on the delivery vans, after which he resumed work on a ball mill grinding graphite and continued at this job until five years before his death, when he ceased work owing to severe shortness of breath. His total exposure to graphite was thus about twenty-two years. When examined in 1946 he was pale and showed slight cyanosis of the lips and some dyspnœa at rest. He said that his cough was severe in the mornings and produced black sputum. A radiograph of his chest showed diffusely scattered shadows of irregular form in both lung fields. The right lung had an appearance of coarse reticulation with irregular patches of coalescence. The whole of the left upper and mid-zones was occupied by denser irregular shadows with coalescence but no definite nodulation. In the left mid-zone was an appearance suggesting a cavity with rather dense walls. Emphysema was seen at both bases and generally throughout both lungs between the denser areas.

This man died on May 7, 1948, and a post-mortem examination was performed for the coroner by Professor P. L. Sutherland. There were old dense pleural adhesions between the lungs and the chest wall on both sides and old adhesions to the diaphragm. Over the lower and outer part of the left lung there were fairly recent adhesions to the chest wall and also recent adhesions to the pericardium. There was fluid in both pleural cavities. The anterior margins of the lungs just met in the middle line. Both lungs were very voluminous and heavy and were emphysematous and odematous. 
The left lung was covered practically all over by thickened pleura: some of the thickening on the lateral lower aspect was fairly recent : the sulcus between the lobes was obliterated by adhesions. The surface of the right lung showed scarred depressed and puckered areas. In both lungs large masses and nodules of dense fibrosis could be felt : on the cut surface of the lung these appeared as jet black areas in some of which there were small cavities. There was no evidence of tuberculosis. Purulent secretion could be expressed from the bronchial tubes on the cut surface. The lung tissue was very black. The glands at the bifurcation of the trachea were enlarged and black but were not hard. The right ventricle of the heart was hypertrophied. The cause of death was silicosis and anthracosis.

A large portion of each lung was received from Professor Sutherland for chemical analysis and microscopical examination. Incineration at $600^{\circ} \mathrm{C}$. had to be repeated several times before all the carbon was oxidized. The ash formed 7.56 per cent., total silica 1.77 per cent., free silica 0.47 per cent., and iron (as $\left.\mathrm{Fe}_{2} \mathrm{O}_{3}\right) 1.07$ per cent. of the dry weight of the lung.

Histological examination showed generalized emphysema and œdema. There was marked pneumoconiosis -showing as linear and radial collagenous fibrosis, and also diffusely scattered nodules of whorled hyaline fibrosis 1 to $3 \mathrm{~mm}$. diameter closely resembling silicotic nodules. There was considerable black pigment, a little of it in phagocytes within alveoli, but mainly in the bands and zones of fibrosis and around the hyaline nodules. A small amount of the black pigment was of irregular rectangular shape up to $30 \mu$ long. Incinerated sections showed that a small proportion of the finer black pigment was iron; the larger irregular particles burnt away and were presumably graphite. Incinerated and acid-treated sections showed a notable dusting of silica visible in polarized light in the areas of fibrosis. The hilar lymph glands showed marked black pigmentation mainly within phagocytes : there were large areas of diffuse collagenous fibrosis in and around the collections of pigmented cells, but no nodular whorled fibrosis.

Other Workers.-Most of the workmen employed at the factory at which Cases 1 and 2 had worked were examined in 1946, and radiographs were taken of their lungs. No men who had less than ten years' exposure to graphite showed any abnormality: all but one of thirteen men whose radiographs showed abnormal shadows had had over twenty years' exposure. Only two of these thirteen were unfit for work (one of these was Case 2), and it was notable that physical disability was on the whole small even in workmen whose radiographs showed marked changes. Brief notes of six of the men follow.

S.A., aged 52 years, had spent twelve years mixing graphite and twenty-two years grinding it. He had severe dyspnœa and was unfit for work. His radiograph showed a diffuse reticular opacity over the whole of both lung fields : the right lung was contracted, especially in its upper part where an irregular patchy opacity at the periphery suggested an area of pleural fibrosis.
G.J., aged 62 years, had worked in the graphite mill for forty-one years. He suffered from morning cough and from dyspnœa on exertion, but was still at work. Radiography showed a large homogeneous area of consolidation in the upper zone of the right lung with confluent opacities extending to the hilum: the midand lower zones of this lung showed pin-head stippling with small patches of confluent opacity. In the left lung was a large homogeneous opacity occupying the apical and infraclavicular region: there was accentuation of the root shadow, and pin-head stippling in the mid-zone.

A.S., aged 44 years, had been grinding graphite for twenty years. He was symptomless, but did not look fit. Radiography showed a diffuse opacity in the upper zone of the right lung with patches of greater density in the first and second interspaces; some mottling by indefinite spots of varying size in the mid- and lower zones, and a general increase in linear markings. In the left lung there was increase in the linear markings with accentuation of the root shadow : linear streaks of increased density fanned outwards from the upper part of the root: there was some indefinite mottling in the mid-zone.

S.J.W., aged 56, was symptomless apart from occasional slight cough after 25 years in the graphite mill. His radiograph showed confluent opacities in the upper zone of the right lung with coarse mottling in the mid-zone : there was patchy opacity with some linear infiltration in the mid-zone of the left lung.

R.W.C., aged 59 years, had worked for twenty-one years in the graphite mill, was symptomless, and felt quite fit. In his right lung radiography showed reticulation and fine mottling in the mid- and lower zones with patchy areas of confluent opacity in the upper zone. In the left lung were a few small scattered nodules varying in size and in sharpness of definition, mostly in the mid-zone.

H.O., aged 56 years, had been grinding graphite for twenty-six years : apart from a very infrequent slight cough he was quite fit. His radiograph showed reticulation (ground-glass appearance) throughout both lungs, most marked in the mid-zones.

The only three men who had been employed for over twenty years at the factory where Case 3 had been employed were examined in 1946. None of them had any physical disability; the radiographs of all three showed reticulation, and one of them showed also nodulation in the right mid-zone.

\section{Animal Experiments}

Under ether anæsthesia intratracheal injections of approximately $50 \mathrm{mg}$. of various samples of graphite from the factory where Cases 1 and 2 had been employed were given to groups of three-months old rats. The animals were killed six to nine months later, and the thoracic organs with the lungs in a state of normal inflation were fixed in formol-saline. Sections were stained with Ehrlich's hæmatoxylin and eosin, with Weigert's elastin stain and van Gieson, and by Wilder's modification of Foot's stain for reticulin fibrils. 
No essential differences were seen between animals given different varieties of graphite. The surface of the lungs had a peculiar whitish appearance that seemed due to fine emphysema. . Pigment was distributed fairly evenly throughout the lungs in small black areas : a few black dots showed in the pleura. Microscopically (figs. 10, 11) there was a marked foreign-body granulomatous reaction around the pigment, some of which appeared as crystalline masses. There were numerous multinucleated giant-cells, and some structures suggesting follicles. Most of the aggregates of pigment with their associated reticulo-endothelial reaction were in the region of lymph junctions alongside vessels or around atria a little distance beneath the pleural surface. There was a fairly marked increase in reticulin fibrils, but only slight collagenous fibrosis.

The histological picture is unlike that produced by similar injections of silica particles on the one hand, and very different from that produced by an artificial, almost pure carbon, graphite on the other.

\section{Discussion}

It has been too readily assumed that graphite led only to a benign form of pneumoconiosis. From the information now available it is possible to say that prolonged exposure to substantial concentrations of graphite dust can produce a pneumoconiosis which, in its advanced stages, may result in complete incapacity for work and can be responsible for death. As with other forms of occupational disease of the lungs, there is a marked variation in susceptibility: some men show gross radiological changes after twenty years' exposure while others show only slight changes after even longer exposure. A striking feature of many cases is the relatively small degree of physical incapacity in relation to the radiological findings, and it appears that this form of pneumoconiosis is often well tolerated until the very late stages of the disease. When increasing dyspnca does lead to incapacity, the further progress seems to be rapid.

The radiological picture is not that of classical silicosis, as the accepted discrete nodulation is seldom seen at any stage. Nor is the histological picture typical of silicosis: nodules resembling those of classical silicosis may be found occasionally but more commonly the picture is one of pigment aggregates with linear or radial fibrosis and an accompanying focal emphysema. In advanced cases massive areas of fibrosis occur without definite nodular formation. In these areas of massive fibrosis necrosis may lead to cavitation. In the small series of workmen reviewed, tuberculosis does not appear to play an important role.

It is evident that graphite pneumoconiosis closely resembles that of coal workers (Cummins and Sladden, 1930; Hart and Aslett, 1942 ; Gough, 1947) both in its histology and in its radiographic appearances. In both graphite workers and coal workers the earliest radiographic signs are reticulation, while the advanced stages show diffuse opacities scattered over both lung fields with the occasional presence of large masses. In both groups of workers necrosis in the centre of a fibrous mass may lead to cavitation and melanoptysis (Dunner and Bagnall, 1946; Stewart and others, 1948) that usually occurs several years after leaving the causative employment : such cavities may not always be visible radiographically. Again in both groups of workers it is quite common to find fairly marked radiological changes in the lungs of men who have little disability. It is uncertain whether the curious bodies described in the lungs of coal workers by Tylecote and Dunn (1931) and by Williams (1934) are the same as the large irregularly rectangular masses of pigment seen in the lungs of graphite workers : they have many similar features.

Graphite pneumoconiosis, then, may be considered as an anthraco-silicosis similar to that of coal workers. For practical purposes graphite can be considered as crystalline carbon mixed with something of the order of 10 per cent. of free silica. It seems to us reasonable to assume that much of the damage to the lungs is produced by this silica, and that we are dealing with a form of silicosis modified by the presence of large amounts of other dust. It is possible also that the very large amounts of dust inhaled may themselves produce some change from sheer overloading of the defensive and drainage mechanisms of the lungs. It is, however, our impression, founded partly on animal work and partly on the examination of workmen, that a pure carbon artificial graphite does not lead to the changes described in this paper.

Conditions of work in the graphite industry vary enormously, and they have on the whole improved greatly in recent years. In view of the findings reported here, graphite can no longer be regarded as a harmless dust. The greatest amount of dust is evolved during the grinding of graphite, which should preferably be done in a totally enclosed mill with a mechanical feed and discharge : failing this, the installation of an adequate exhaust ventilation system is necessary. At all times exposure should be limited to that necessarily entailed in the feeding and discharge of the mill, and an efficient dust respirator is desirable whenever exposure to graphite dust is inevitable. 


\section{Summary}

A brief description is given of the industrial uses of natural graphite, which is a crystalline carbon mixed with up to 10 per cent. of free silica and with other minerals. The greatest dust risk occurs in the grinding of natural graphite, and evidence is presented to show that this can lead to disabling and fatal pneumoconiosis. Marked changes in the lungs are, however, seldom seen in grinders who have less than twenty years' exposure. The results of three autopsies and some of the findings in other workmen are given, together with the results of some experiments on animals. The radiological and histological findings are similar to those found in South Wales coal workers.

We are grateful to Dr. N. T. Whitehead and Prof. P. L. Sutherland for their notes on the post-mortem findings in Cases 2 and 3 respectively, to Dr. J. C. Lees for the blocks of the lungs of Case 1, and to Dr. J. Glover for generous samples of graphite and carbon black. It gives us pleasure to record the helpfulness of Dr. A. I. G. McLaughlin and others in the Factory Department of the Ministry of Labour and National Service. The photographs were made by Mr. A. W. Collins, F.I.M.L.T.

\section{REFERENCES}

Cummins, S. L., and Sladden, A. F. (1930). J. Path. Bact., 33, 1095 Dassanayake, W. L. P. (1948). Brit. J. industr. Med., 5, 141 .

Dunner, L. (1945). Brit. J. Radiol., 18, 33

, and Bagnall, D. J. T. (1946). Ibid., 19, 165.

Geever, E. F. (1947). Amer. J. med. Sci., 214, 292.

Gough, J. (1947). Occupat. Med., 4, 86.

Hart, P. d'A., and Aslett, E. A (1942). Spec. Rep. Ser. Med. Res. Counc., London. No. 243

Jones, W. R. (1943). “ Minerals in Industry." London. p. 57.

Koopmann, H. (1924). Virchows Arch., 253, 423.

Stewart, A, and others (1948), Brit. J. industr. Med, 5, 120

Stewart, M. J and Faulds J.S (1934). J. Path. Bact 39, 233

Trostel,L. J., and Wynne, D. J. (1940). Amer. Ceramic Soc. J., 23, 18.

Trostel, L. J., and Wynne, D. J. S. (1931). Amer. Ceramcet, 2, 632.

Tylecote, F. E., and Dunn, J. S. (1931 